\title{
TRANSIENT HEAT FLOW FROM A THIN CIRCULAR DISK - SMALL-TIME SOLUTION
}

\author{
J. H. BLACKWELL
}

(Received 4 February 1970; revised 21 April 1970)

Communicated by A. F. Pillow

Summary. In a previous paper [1] an approximate analytical solution, useful for large times, was obtained for the transient heat flow from a thin circular disk held at constant temperature and immersed in an infinite medium. In the present work a first approximation has been found for the complementary "small-time" solution and the details of this solution examined. Some numerical calculations are included.

\section{Introduction}

Several years ago the author and a colleague published a paper on transient heat flow from oblate and prolate spheroids held at constant temperature and immersed in a medium of infinite extent [1].

The circular disk of vanishingly small thickness was treated as a limiting case of the oblate spheroid and, in fact, the need for a solution to the experimental problem of the disk provided the original motivation for the problem. It was noted in the paper that the constant-temperature disk problem is much more difficult than that of a disk generating heat at a known rate.

A deficiency of the solution in the 1964 paper was that the formal expansion obtained was suitable for calculation only when the dimensionless time parameter was large compared with unity. All attempts at the time to obtain a complementary "small-time" solution failed.

Recently the writer, helped by the view-point of singular perturbation theory, has been able to obtain a formal first approximation to a "small-time" solution. This solution is discussed below for the circular disk - the method may be readily extended to the general spheroids but for simplicity and since the experimental significance of the more general cases is probably slight, the latter are not included.

In outline the method is as follows. The Fourier heat conduction equation in oblate spheroidal co-ordinates is Laplace-transformed with respect to time. In the resulting Helmholtz equation the parameter $q=p^{\frac{1}{2}} a / k^{\frac{1}{2}}$ (where ' $p$ ' is the transform variable, ' $a$ ' the disk radius, and ' $k$ ' the thermal diffusivity) is treated as large and the spheroidal co-ordinates "stretched" in terms of it. An expansion 
in inverse powers of $q$ is then assumed for the stretched equation and boundary conditions, and successive subsidiary problems result. The first-approximate problem is then solved and inverse transformed.

Implicit throughout is the assumption that inverse transformation of an approximate solution for large $p^{\frac{1}{2}} a / k^{\frac{1}{2}}$ yields an approximate solution for small $k t / a^{2}(2)$.

Before developing the present method an attempt was made without success to solve the problem in cylindrical co-ordinates using the technique of dual integral equations.

\section{Analysis}

The oblate spheroidal co-ordinates $(\varepsilon, \eta)$ used are related to ordinary cylindrical co-ordinates by

$$
\begin{array}{lrl}
r=a\left[\left(1+\varepsilon^{2}\right)\left(1-\eta^{2}\right)\right]^{\frac{1}{2}} & 0 \leqq \varepsilon, \\
z=a \varepsilon \eta & -1 \leqq \eta \leqq 1, \\
\phi=\phi & 0 \leqq \phi \leqq 2 \pi
\end{array}
$$

and, with axial symmetry, the heat conduction equation and boundary conditions in these co-ordinates are:-

$$
\begin{aligned}
& \frac{1}{a^{2}\left(\varepsilon^{2}+\eta^{2}\right)}\left[\frac{\partial}{\partial \varepsilon}\left\{\left(\varepsilon^{2}+1\right) \frac{\partial \theta}{\partial \varepsilon}\right\}+\frac{\partial}{\partial \eta}\left\{\left(1-\eta^{2}\right) \frac{\partial \theta}{\partial \eta}\right\}\right]=\frac{1}{k} \frac{\partial \theta}{\partial t} \\
& 0<\varepsilon ;-1 \leqq \eta \leqq 1 ; t>0
\end{aligned}
$$

with

$$
\begin{array}{rrrrrr}
\varepsilon & =0 & \theta=\theta_{0} & -\leqq \eta \leqq 1 ; & t>0 \\
\eta=0 & \frac{\partial \theta}{\partial \eta}=0 & 0<\varepsilon ; & t>0 \\
t=0 & \theta=0 & 0<\varepsilon ; & -1 \leqq \eta \leqq 1
\end{array}
$$

where

$\varepsilon, \eta, t$ are the "radial" and "angular" co-ordinates and the time insert $\theta(\varepsilon, \eta, t), k$ are the temperature and thermal diffusivity of the external medium semi-color $\theta_{0}, a$ are the temperature and radius of the disk, $\varepsilon=0$.

Taking the Laplace transform of equation (1) and boundary conditions with respect o time we obtain

$$
\begin{aligned}
\frac{\partial}{\partial \varepsilon}\left\{\left(\varepsilon^{2}+1\right) \frac{\partial \Theta}{\partial \varepsilon}\right\}+\frac{\partial}{\partial \eta}\left\{\left(1-\eta^{2}\right) \frac{\partial \Theta}{\partial \eta}\right\}=q^{2}\left(\varepsilon^{2}+\eta^{2}\right) \Theta & \\
0 & <\varepsilon ; \quad-1 \leqq \eta \leqq 1
\end{aligned}
$$


with

$$
\begin{array}{llll}
\varepsilon=0 & \Theta=\frac{\theta_{0} a^{2}}{k q^{2}} & -1 \leqq \eta \leqq 1 \\
\eta=0 & \frac{\partial \Theta}{\partial \eta}=0 & 0<\varepsilon
\end{array}
$$

where $\Theta(\varepsilon, \eta, p)=L_{t} \theta(\varepsilon, \eta, t)$ and $q=(p / k)^{\frac{1}{2}} a$.

The problem of (5), (6), (7) can be solved exactly in terms of spheroidal wave-functions and reference to this was made in the original paper [1]. In the latter case, the algebraic complexity of manipulating and inverting these functions led us to abandon the approach in favour of the less direct, but algebraically simpler one employed.

With the present work, where the basic requirement is to find an approximate solution of (5), (6), (7) for large values of the parameter ' $q$ ', the argument for finding an alternative to the exact solution seems, if anything, stronger. The employment of a Watson transformation, as suggested by the referee, would undoubtedly help in reducing the exact solution but, in the writer's opinion, the method below will lead much more quickly and simply to a first-approximate solution of the original problem described by (1), (2), (3), (4).

Since ' $q$ ' is to be regarded as large in equation (5), when we divide that equation by ' $q$ ', the highest order (in this instance, all) derivatives are multiplied by the small parameter ' $1 / q^{2}$ '. This suggests that we adopt the viewpoint of singular perturbation theory [3] [4] and look for an "inner" or "boundarylayer'" type solution. This will involve:

(a) "stretching" the variables, $\varepsilon, \eta$ so that in the limit $1 / q \rightarrow 0$ the highest derivatives in the "stretched" variables will be retained

(b) assuming an expansion for the solution in a series of functions of the "stretched" variables, whose coefficients form an appropriate asymptotic sequence in powers of $1 / q$.

The appropriate stretching is given by

$$
\begin{aligned}
& v=q^{\frac{1}{2}} \varepsilon \\
& \omega=q^{\frac{1}{2}} \eta
\end{aligned}
$$

Then in terms of $v, \omega(5),(6),(7)$ becomes

$$
\frac{\partial}{\partial v}\left\{\left(1+\frac{v^{2}}{q}\right) \frac{\partial \Theta}{\partial v}\right\}+\frac{\partial}{\partial \omega}\left\{\left(1-\frac{\omega^{2}}{q}\right) \frac{\partial \Theta}{\partial \omega}\right\}=\left(v^{2}+\omega^{2}\right) \Theta
$$

with

$$
\begin{array}{lll}
v=0 & \Theta=\frac{\theta_{0} a^{2}}{k q^{2}} \\
\omega=0 & \frac{\partial \Theta}{\partial \omega}=0
\end{array}
$$


Now assume an expansion for $(\Theta)$ in inverse powers of $q$

$$
=\frac{1}{q^{2}} \Theta_{1}(v, \omega)+\frac{1}{q^{3}} \Theta_{2}(v, \omega)+\cdots
$$

substitute in (8), (9), (10) and equate coefficients of like powers of $1 / q$. We obtain:

(i)

$$
\Theta_{1 v}+\Theta_{1 \omega \omega}-\left(v^{2}+\omega^{2}\right) \Theta_{1}=0
$$

with

$$
\begin{array}{ll}
v=0 & \Theta_{1}=\frac{\theta_{0} a^{2}}{k} \\
\omega=0 & \Theta_{1 \omega}=0
\end{array}
$$

(ii)

$$
\Theta_{2 v v}+\Theta_{2 \omega \omega}-\left(v^{2}+\omega^{2}\right) \Theta_{2}=-\frac{\partial}{\partial v}\left(v^{2} \Theta_{1 v}\right)+\frac{\partial}{\partial \omega}\left(\omega^{2} \Theta_{1 \omega}\right)
$$

with

$$
\begin{aligned}
v & =0 & \Theta_{2} & =0 \\
\omega & =0 & \Theta_{2 \omega} & =0
\end{aligned}
$$

and so on.

The problem (i) can be solved in closed form in terms of familiar transcendental functions. In the method used by the writer, the independent variables are first transformed to $x, \phi$ defined by

$$
x=\frac{1}{2}\left(v^{2}+\omega^{2}\right) \quad \phi=\tan ^{-1}(\omega / v) .
$$

The boundary-value problem in the new independent variables is then solved by a Kontorovitch-Lebedev transform [5] and we obtain

$$
\Theta_{1}=\frac{2}{\pi}\left(\frac{\theta_{0} a^{2}}{k}\right) \int_{0}^{\infty} K_{i \alpha}(x) \frac{\cosh \frac{1}{2} \pi \alpha \cosh 2 \phi \alpha}{\cosh \pi \alpha} d \alpha
$$

Much tedious reduction of this integral leads to

$$
\Theta_{1}=\frac{\theta_{0} a^{2}}{2 k},\left[e^{-v \omega} \operatorname{erfc}\left(\frac{v-\omega}{\sqrt{ } 2}\right)+e^{v \omega} \operatorname{erfc}\left(\frac{v+\omega}{\sqrt{ } 2}\right)\right]
$$

Hence the first approximation to $\Theta$ is

$$
\Theta^{(1)}=\frac{\theta_{0}}{2 p}\left[e^{-v \omega} \operatorname{erfc}\left(\frac{v-\omega}{\sqrt{ } 2}\right)+e^{v \omega} \operatorname{erfc}\left(\frac{v+\omega}{\sqrt{ } 2}\right)\right]
$$

Having obtained $\Theta_{1}$ it is possible, in principle, to then solve problem (ii) and so on. 
In fact, the solution of even problem (ii) is extremely complex and, in the writer's opinion, the usefulness of the result does not justify the labour which would be required in obtaining it. Let us now consider the inverse transformation of equation (18). Rewriting that equation, we have

$$
\Theta^{(1)}=\frac{\theta_{0}}{2 p}\left[e^{-q \varepsilon \eta} \operatorname{erfc}\left\{\frac{q^{\frac{1}{2}}(\varepsilon-\eta)}{\sqrt{ } 2}\right\}+e^{q \varepsilon \eta} \operatorname{erfc}\left\{\frac{q^{\frac{1}{2}}(\varepsilon+\eta)}{\sqrt{2}}\right\}\right]
$$

or

$$
\begin{array}{r}
\Theta^{(1)}=\frac{\theta_{0}}{2 p} \exp \left\{-\frac{q\left(\varepsilon^{2}+\eta^{2}\right)}{2}\right\}\left[\exp \frac{q(\varepsilon-\eta)^{2}}{2} \operatorname{erfc}\left\{\frac{q^{\frac{1}{2}}(\varepsilon-\eta)}{\sqrt{ } 2}\right\}\right. \\
\left.+\exp \left\{\frac{q(\varepsilon+\eta)^{2}}{2}\right\} \operatorname{erfc}\left\{\frac{q^{\frac{1}{2}}(\varepsilon+\eta)}{\sqrt{ } 2}\right\}\right]
\end{array}
$$

By standard methods, we can invert (19) and express the result as a real infinite integral which can be evaluated numerically. However, in view of the fact that the solution (19) is itself just the leading term in an expansion of $\Theta$ in powers of $1 / q$, it is probably more useful to proceed with further expansion of (19) and make approximate inverse transformation. The quite complicated integral resulting from 'exact' inversion of [19] has been derived by the writer but is not, for the reason just stated, included in this paper. To avoid confusion we shall assume in the following that $\eta \geqq 0$. Results for $\eta \geqq 0$ follow at once from symmetry.

Two cases can be distinguished:

Case (i) $\eta, \varepsilon$ not approximately equal;

Case (ii) $\eta, \varepsilon$ approximately equal i.e. $|\varepsilon-\eta| \ll \varepsilon$.

Not surprisingly, neither solution is valid in the limit of points on the disk periphery $(\varepsilon=\eta=0)$, where there is an impulsive redistribution of temperature at $t=0$.

We shall require the following transform pairs [6]

$$
\begin{aligned}
& L^{-1} \frac{e^{-A p^{\frac{1}{2}}}}{p^{\frac{\pi}{4}}}=\frac{1}{\pi}\left(\frac{A}{2 t}\right)^{\frac{1}{2}} e^{-A^{2} / 8} K_{\frac{1}{4}}\left(\frac{A^{2}}{8 t}\right) \\
& L^{-1} \frac{e^{-A p^{\frac{1}{2}}}}{p^{\frac{1}{t}}}=\frac{1}{\pi}\left(\frac{A^{3}}{2 t}\right)^{\frac{1}{2}} e^{-A^{2} / 8 t}\left[K_{\frac{3}{4}}\left(\frac{A^{2}}{8 t}\right)-K_{\frac{1}{4}}\left(\frac{A^{2}}{8 t}\right)\right]
\end{aligned}
$$

Case (i): $\eta, \varepsilon$ not approximately equal.

In this case we can treat the arguments of the function in the square bracket of equation (19b) as large in magnitude and replace the functions by the dominant terms of their asymptotic expansions

For $x$ with large positive real part

$$
\operatorname{erfc} x \simeq \pi^{-\frac{1}{2}} e^{-x^{2}} / x
$$




$$
\operatorname{erfc}(-x)=2-\operatorname{erfc} x \simeq 2-\pi^{-\frac{1}{2}} e^{-x^{2}} / x
$$

Hence we have

$$
\text { (a) } 0 \leqq \eta<\varepsilon: \Theta^{(1)} \simeq \sqrt{\frac{2}{\pi}} \frac{\theta_{0}}{p q^{\frac{1}{2}}}\left(\frac{\varepsilon}{\varepsilon^{2}-\eta^{2}}\right) \exp \left\{-q \frac{\left(\varepsilon^{2}+\eta^{2}\right)}{2}\right\}
$$

(b) $0 \leqq \varepsilon<\eta: \Theta^{(1)} \simeq \theta_{0}\left[\frac{e^{-q \varepsilon \eta}}{p}-\sqrt{\frac{2}{\pi}} \frac{\theta_{0}}{p q^{\frac{3}{2}}}\left(\frac{\varepsilon}{\eta^{2}-\varepsilon^{2}}\right) \exp \left\{-q \frac{\left(\varepsilon^{2}+\eta^{2}\right)}{2}\right\}\right]$

Then inverting, using [21]

$$
\text { (a) } 0 \leqq \eta<\varepsilon: \theta^{(1)}=L^{-1} \Theta^{(1)} \simeq(2 \pi)^{-\frac{\varepsilon}{2}} \theta_{0}\left\{\frac{\varepsilon\left(\varepsilon^{2}+\eta\right)^{\frac{2}{2}}}{\varepsilon^{2}-\eta^{2}}\right\}
$$

$$
\times T^{-\frac{1}{2}} e^{-X}\left\{K_{\frac{3}{4}}(X)-K_{\frac{1}{4}}(X)\right\}
$$

(b) $0 \leqq \varepsilon<\eta: \theta^{(1)} \simeq \theta_{0}\left[\operatorname{erfc}\left(\frac{z}{2 a T^{\frac{1}{2}}}\right)-(2 \pi)^{-\frac{z}{2}}\right.$

$$
\left.\times\left\{\frac{\varepsilon\left(\varepsilon^{2}+\eta^{2}\right)^{\frac{3}{2}}}{\eta^{2}-\varepsilon^{2}}\right\} T^{-\frac{1}{2}} e^{-X}\left\{K_{\frac{3}{4}}(X)-K_{f}(X)\right\}\right]
$$

where

$$
\begin{gathered}
T=\frac{k t}{a^{2}} \\
X=\frac{\left(\varepsilon^{2}+\eta^{2}\right)^{2}}{32 T}
\end{gathered}
$$

Since $T$ is small and hence $X$ large we can simplify these results further by introducing the asymptotic expansions of the Bessel functions

$$
K_{\frac{3}{4}}(X)-K_{\frac{1}{4}}(X) \sim \sqrt{\frac{\pi}{2 X}} e^{-X}\left[1+\frac{5}{32 X}+\cdots-\left(1-\frac{3}{32 X}+\cdots\right)\right]
$$

$$
\sim \sqrt{\frac{\pi}{2 X}} e^{-x}\left[\frac{1}{4 X}+O\left(\frac{1}{X^{2}}\right)\right]
$$

Substituting the dominant term, we have finally

$$
\begin{aligned}
& \text { (a) } 0 \leqq \eta<\varepsilon: \theta^{(1)} \simeq 2^{7 / 2} \pi^{-1}\left[\frac{\varepsilon \theta_{0}}{\left(\varepsilon^{2}+\eta^{2}\right)^{3 / 2}\left(\varepsilon^{2}-\eta^{2}\right)}\right] T e^{-2 X} \\
& \text { (b) } 0 \leqq \varepsilon<\eta: \theta^{(1)} \simeq \theta_{0}\left[\operatorname{erfc}\left(\frac{z}{2 a T^{\frac{1}{2}}}\right)\right.
\end{aligned}
$$

$$
\left.-2^{7 / 2} \pi^{-1} \frac{\varepsilon}{\left(\varepsilon^{2}+\eta^{2}\right)^{3 / 2}\left(\eta^{2}-\varepsilon^{2}\right)} T e^{-2 X}\right]
$$


The limiting solution on the plane surrounding the disk $(\eta=0)$ is, from (27)

$$
\theta^{(1)} \simeq 2^{7 / 2} \pi^{-1} \frac{\theta_{0}}{\varepsilon^{4}} T e^{-2 X_{0}}=2^{7 / 2} \pi^{-1} \frac{\theta_{0}}{\left(r^{2} / a^{2}-1\right)^{2}} T e^{-2 x_{n}}
$$

where

$$
X_{0}=\frac{\left(r^{2} / a^{2}-1\right)^{2}}{32 T}
$$

On the axis of the disk above the plane, $\eta=1$, and $\varepsilon=z / a$. Thus on the axis, from equations (27), (28)

$$
\text { (a) } 1<\varepsilon: \theta^{(1)} \simeq 2^{7 / 2} \pi^{-1} \theta_{0} \frac{a^{4} z}{\left(z^{2}+a^{2}\right)^{3 / 2}\left(z^{2}-a^{2}\right)} T e^{-2 x}
$$

(b) $0 \leqq \varepsilon<1: \theta^{(1)} \simeq \theta_{0}\left[\operatorname{erfc}\left(\frac{z}{2 a T^{\frac{1}{2}}}\right)\right.$

$$
\left.-2^{7 / 2} \pi^{-1} \frac{a^{4} z}{\left(z^{2}+a^{2}\right)^{3 / 2}\left(a^{2}-z^{2}\right)} T e^{-2 X}\right]
$$

where

$$
X=\frac{1}{32 T}\left(1+z^{2} / a^{2}\right)^{2} .
$$

It will be noted that the approximate solutions (27), (28) indeed satisfy the initial and boundary conditions at $t=0, \varepsilon=0, \eta=0$, except when $\varepsilon \simeq \eta \simeq 0$. A rough estimate of the range of validity of (27) and (28) is

$$
\frac{1}{X}=\frac{32 T}{\left(\varepsilon^{2}+\eta^{2}\right)^{2}} \ll 1
$$

Case (ii): $|\varepsilon-\eta| \ll \varepsilon$,

In the immediate neighbourhood of $\eta=\varepsilon$ we treat the argument

$$
\left|q^{\frac{1}{2}}(\varepsilon-\eta)\right|
$$

as small. We expand the first term in equation [19b] in its ascending series expansion in

$$
\frac{q^{\frac{1}{2}}(\varepsilon-\eta)}{\sqrt{ } 2}
$$

and retain the dominant terms, that is,

$$
\exp \left\{\frac{q(\varepsilon-\eta)^{2}}{2}\right\} \operatorname{erfc}\left\{\frac{q^{\frac{1}{2}}(\varepsilon-\eta)}{\sqrt{2}}\right\} \approx 1-\sqrt{\frac{2}{\pi}} q^{\frac{1}{2}}(\varepsilon-\eta) .
$$

Treating the second term as in case (1) and substituting in (19b) we get 
(33) $\Theta^{(1)} \simeq \frac{\theta_{0}}{2 p} \exp \left\{\frac{-q\left(\varepsilon^{2}+\eta^{2}\right)}{2}\right\}\left[1-\sqrt{\frac{2}{\pi}} q^{\frac{1}{2}}(\varepsilon-\eta)+\sqrt{\frac{2}{\pi}} \frac{1}{q^{\frac{1}{2}}(\varepsilon+\eta)}\right]$.

Then inverting, using (20) and (21)

$$
\begin{aligned}
\theta^{(1)}=L^{-1} \Theta^{(1)} & \simeq \theta_{0}\left[\frac{1}{2} \operatorname{erfc}(2 X)^{\frac{1}{2}}+\frac{\left(\varepsilon^{2}+\eta^{2}\right)^{\frac{1}{2}}}{2(\varepsilon+\eta)}(2 \pi)^{-\frac{3}{2}} T^{-\frac{1}{2}} e^{-X}\right. \\
& \left.\times\left[\left(\varepsilon^{2}+\eta^{2}\right) K_{\frac{2}{2}}(X)-\left(3 \varepsilon^{2}-\eta^{2}\right) K_{\frac{1}{4}}(X)\right]\right]
\end{aligned}
$$

As before, introducing the dominant terms in the asymptotic expansions of the Bessel functions we get

$$
\theta^{(1)} \simeq \theta_{0}\left[\frac{1}{2} \operatorname{erfc}(2 X)^{\frac{1}{2}}-2^{\frac{1}{2}} \pi^{-1} \frac{\varepsilon-\eta}{\left(\varepsilon^{2}+\eta^{2}\right)^{\frac{1}{2}}} e^{-2 X}\right]
$$

It is quickly seen that to the order of the approximation used in (35), we may replace

$$
\frac{\varepsilon^{2}+\eta^{2}}{2} \text { by } \varepsilon \eta \text { i.e. by } z / a
$$

Thus we can rewrite (35)

$$
\theta^{(1)} \simeq \theta_{0}\left[\frac{1}{2} \operatorname{erfc}\left(\frac{z}{2 a T^{\frac{1}{2}}}\right)-2^{\frac{1}{2}} \pi^{-1} \frac{(\varepsilon-\eta) a^{\frac{1}{2}}}{z^{\frac{1}{2}}} \exp \left(-\frac{z^{2}}{4 a^{2} T}\right)\right]
$$

When $\varepsilon=\eta$ i.e. on the sphere radius 'a', centre at the origin, the temperature in this approximation is just half what it would be if the whole plane $z=0$ were held at temperature $\theta_{0}$.

With similar reasoning to that of case (i), we expect the solution of equation (35) to be useful for

$$
\frac{8 T}{\varepsilon^{4}} \ll 1
$$

\section{Numerical illustration}

Some numerical values of $\theta / \theta_{0}$ for the case $\eta=0$ are shown in Table 1 .

The table contains values calculated from the large-time solution of the original paper [1], shown in italics, and, in ordinary type, values calculated from the small-time solution of equation (29).

For certain pairs near the limit of validity of one or both of the approximations values obtained from each are shown. In Figures $1,2 \theta / \theta_{0}$ is plotted against $T$ for $\varepsilon=1,2$, the solid curves representing the values given by the two approximations and the dotted curve a smooth-curve interpolation in the region where neither approximation is valid. 

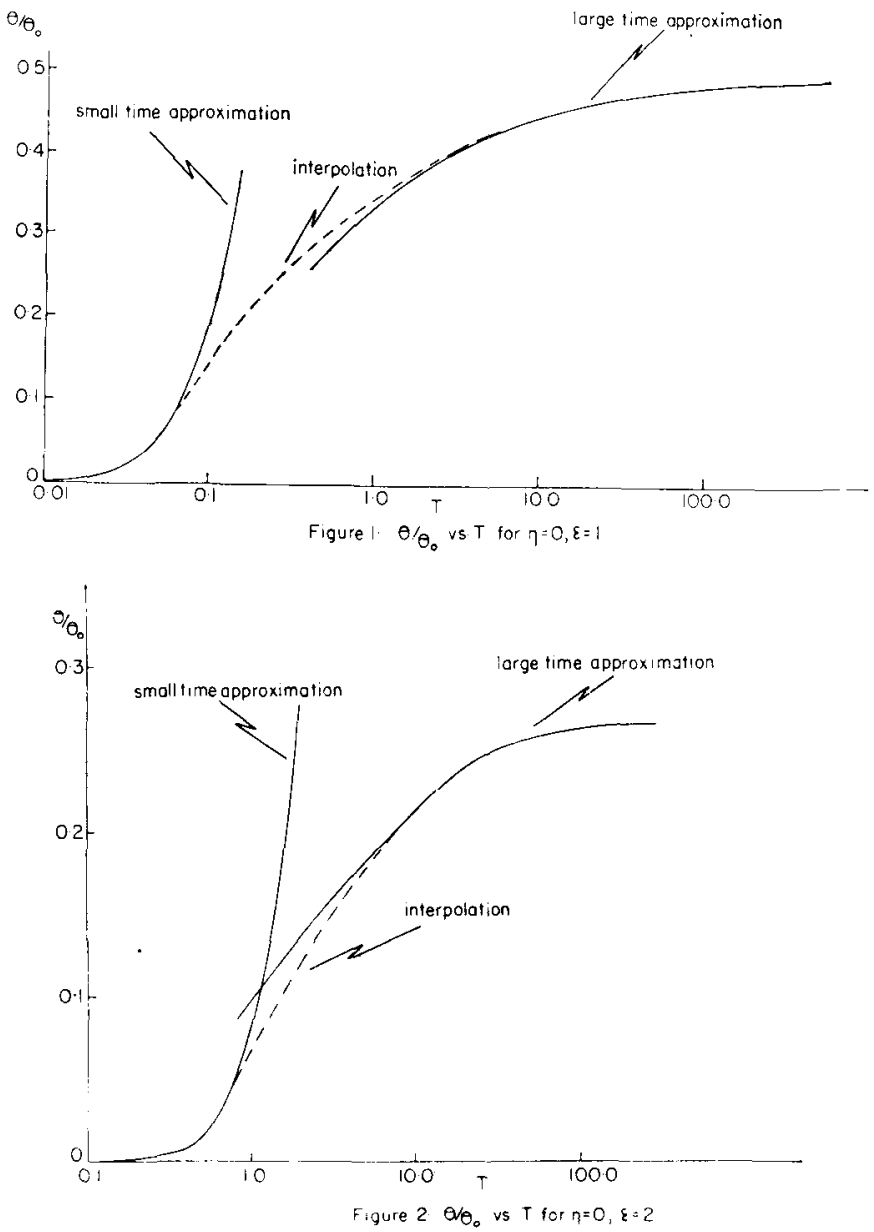

\section{Discussion}

Although the usefulness of small-time approximate solutions is usually not as great as those for large-times, the present work completes the unifinshed work of the earlier paper on a classical problem of transient heat conduction.

An exact solution is closed form of the complete problem seems impossibly difficult to obtain, and, if required, the obvious way to improve on these results by a full numerical treatment.

\section{Acknowledgements}

The writer wishes to acknowledge gratefully a grant-in-aid from the National Research Council of Canada, during the tenure of which this work has been carried out. 
TABLE 1

Values of $\theta / \theta_{0}$ for $\eta=0$

\begin{tabular}{|c|c|c|c|c|c|}
\hline$T \varepsilon$ & 1 & 2 & 3 & 4 & 6 \\
\hline 25 & 0.464 & 0.244 & 0.148 & $\begin{array}{l}0.098 \\
0.185\end{array}$ & 0.0027 \\
\hline 4 & 0.412 & 0.169 & $\begin{array}{l}0.062 \\
0.050\end{array}$ & 0.0010 & $<10^{-10}$ \\
\hline 1 & 0.332 & $\begin{array}{l}0.098 \\
0.083\end{array}$ & $<10^{-3}$ & $<10^{-8}$ & \\
\hline 0.5 & $\begin{array}{l}0.278 \\
1.589\end{array}$ & 0.015 & & & \\
\hline 0.1 & 0.193 & $<10^{-5}$ & & & \\
\hline 0.05 & 0.052 & & & & \\
\hline 0.03 & 0.013 & & & & \\
\hline 0.01 & $<10^{-4}$ & & & & \\
\hline
\end{tabular}

\section{References}

[1] E. J. Norminton and J. H. Blackwell, 'Transient heat flow from constant temperature spheroids and the thin circular disk', Quart. J. of Mech. and App. Math. 17, pt. 1, (1964), 65-72.

[2] H. S. Carslaw and J. C. Jaeger, Operational methods in Applied Mathematics, 2nd ed. (Oxford University Press, London, 1948), 276-279.

[3] J. D. Cole, Perturbation Methods in Applied Mathematics (Blaisdell, Waltham, Mass. 1958).

[4] M. van Dyke, Perturbation Methods in Fluid Mechanics. (Academic Press, New York, 1964).

[5] A. Erdélyi, W. Magnus, F. Oberhettinger. and F. G. Tricomi, Tables of Integral Transforms, Vol. II, (McGraw-Hill, New York, 1954), 175.

[6] H. S. Carslaw and J. C. Jaeger, Condition of heat in solids, 2nd ed. (Oxford University Press, Oxford, 1959), 495.

Department of Applied Mathematics

University of Western Ontario

Canada 